\title{
Unilateral choroidal tuberculoma as an initial manifestation of presumed ocular tuberculosis: a case report
}

Tuberculoma coroidal unilateral como manifestação inicial de tuberculose ocular presumida: relato de caso

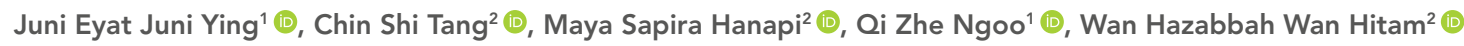

Keywords:

Tuberculoma; Tuberculosis, ocular; Uveitis, posterior

Descritores:

Doenças da coroide/ diagnóstico; Tuberculoma; Tuberculose ocular; Uveíte posterior

Received on: Feb 22, 2021

Accepted on: Apr 21, 2021

Corresponding author: Ngoo Qi Zhe Department of Ophthalmology, School of Medical Sciences, Health Campus, Universiti Sains Malaysia, 16150 - Kota Bharu, Kelantan, Malaysia E-mail: henryzhe@usm.my

Institution: Universiti Sains Malaysia, Kota Bharu, Kelantan, Malaysia.

Conflict of interest: no conflict of interest.

Financial support: the authors received no financial support for this work.

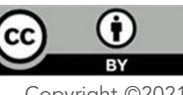

Copyright (C2021

\section{ABSTRACT}

Choroidal tuberculomas are present in patients with ocular tuberculosis. They usually occur in a patient with previous history of tuberculosis, and are rarely the initial presentation, with no prior systemic manifestations. We present a patient with unilateral choroidal tuberculoma as the initial presentation of presumed ocular tuberculosis, which enabled earlier initiation of treatment.

\section{RESUMO}

Os tuberculomas de coroide apresentam-se em pacientes com tuberculose ocular. Geralmente, ocorrem em indivíduos com história prévia de tuberculose e raramente têm apresentação inicial sem manifestações sistêmicas anteriores. Relatamos o caso de um paciente com tuberculoma de coroide unilateral com apresentação inicial de tuberculose ocular presumida, permitindo o início mais precoce do tratamento. 


\section{INTRODUCTION}

Choroidal tuberculomas are large single lesions that usually occur in patients with a past history of tuberculosis (TB). ${ }^{(1)}$ They commonly occur in young patients with a prolonged course of disease. Unlike their counterpart choroidal tubercles, they are usually better defined due to less surrounding retinal edema. They are usually located on the posterior pole, particularly in the parafovea region. ${ }^{(2)}$ It is not usual for choroidal tuberculoma to be the first presentation in patient with ocular TB without systemic presentation.

Hence, we reported this case, who presented with solitary choroidal tuberculoma and was treated as presumed ocular TB. We were given consent for the above picture and details of patient to be disclosed in this case report.

\section{CLINICAL CASE}

A previously well 27-year-old Malay lady presented with right eye blurring of vision for 6 weeks. It was associated with central scotoma. She had no complaints of eye redness, floaters, photophobia and flashes of light. Patient reported having with pulmonary TB patient six months ago, prior to presentation. No other constitutional symptoms noted. Patient had no family history of malignancy or blood dyscrasia.

Upon initial examination, vision over right eye was $6 / 60$, her left eye was $6 / 6$. On anterior segment examination of the right eye, there were no iris nodules or broad based posterior synechiae. The lens was clear. The anterior segment examination of the fellow eye was normal. Fundus examination of right eye revealed choroidal tuberculoma of approximately three disc-diameter size over the inferotemporal arcade, just inferior to the fovea with minimal surrounding retinal edema (Figures 1 and 2). The optic disc was normal. Scattered retinal hemorrhages were seen overlying the choroidal tuberculoma. There were no obvious retinitis or choroiditis. The posterior segment examination of the left eye was normal. The intraocular pressure was normal in both eyes. B-scan ultrasonography of the right eye revealed a choroidal mass showing high reflectivity in the anterior border, but no orbital shadowing (Figure 3). Fundus fluorescein angiography (FFA) of the eye was done, showing a hypofluorescent lesion during the early phase that became hyperfluorescent towards the late phase (Figures 4 and 5). Full blood count was normal. Erythrocyte sedimentation rate was elevated at $30 \mathrm{~mm}$ /hour. Mantoux test was $17 \mathrm{~mm}$. Other infective screening tests, including Toxoplasma, VDRL for

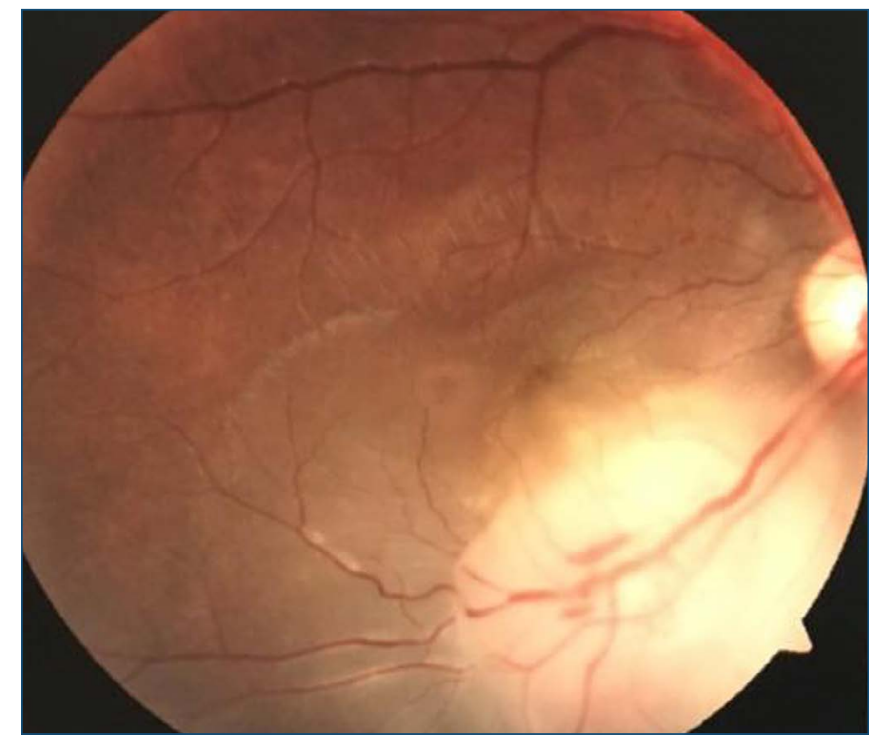

Figure 1. Choroidal tuberculoma at the inferotemporal arcade, of approximately three disc-diameter size.

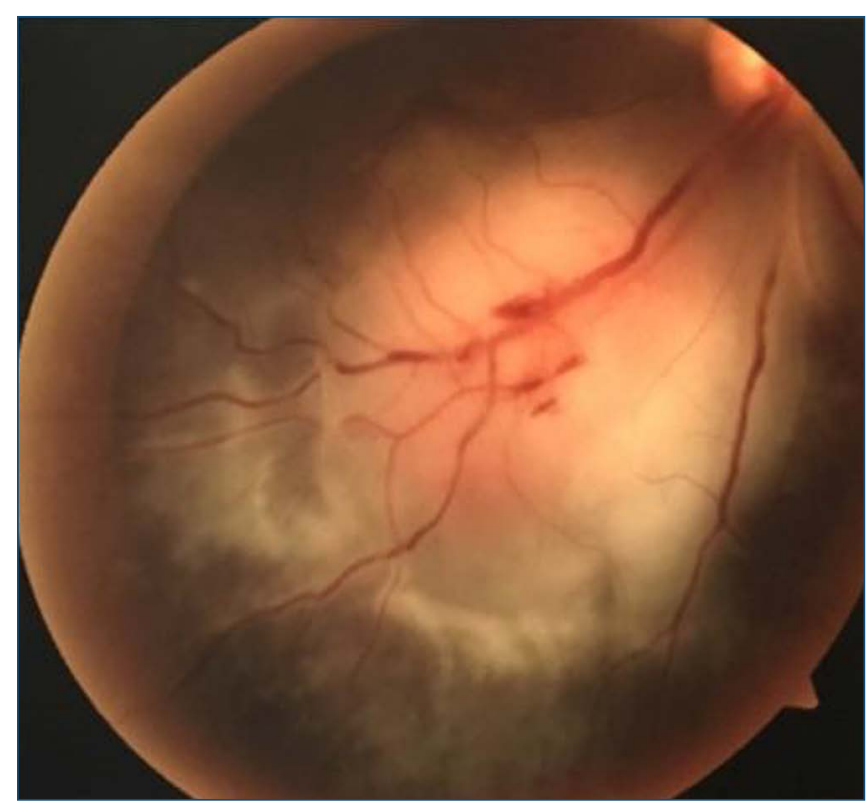

Figure 2. A magnified image of the tuberculoma with surrounding retinal edema, and overlying retinal hemorrhages.

syphilis serology, herpes simplex virus (HSV) and cytomegalovirus serology, were negative. The connective tissue disease screening was also negative. A diagnosis of posterior uveitis secondary to presumed ocular TB in the right eye was made. Brain and orbital computed tomography was performed and there was no involvement of brain meninges and parenchyma. There was a focal enhancing nodular thickening at posterior retina of the right globe measuring $4.5 \mathrm{~mm}$. Patient refused lumbar puncture to confirm no central nervous system involvement. Patient was co-managed by the infectious disease team and the diagnosis of presumed ocular TB was confirmed. Subsequently, 8 weeks of induction 


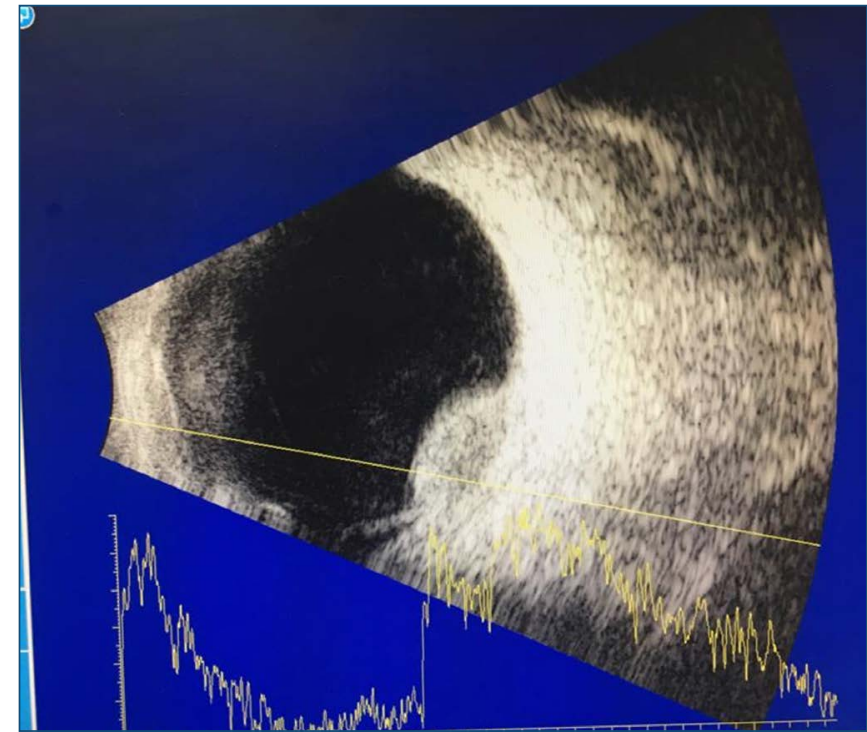

Figure 3. B-scan ultrasonography of the right eye showing choroidal mass with high reflectivity but no shadowing.

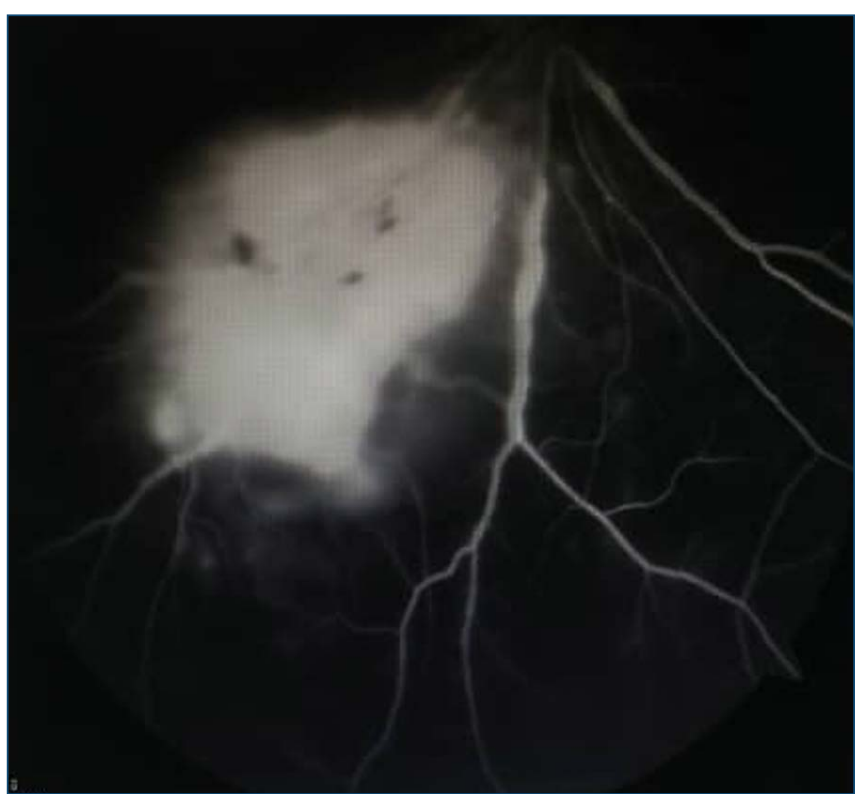

Figure 4. FFA showed increasing hyperfluorescent towards the venous phase with retinal perivasculitis.

systemic antitubercular treatment was initiated, followed by 7 months of maintenance therapy. One week after initiating antitubercular therapy, oral prednisolone at $0.5 \mathrm{mg} / \mathrm{kg}$ was started, to reduce inflammation caused by the primary insult.

Her subsequent follow-up, 4 weeks after initiating treatment, showed an improvement of vision to 6/18 in her right eye. Anterior chamber was quiet. Intraocular pressure was normal. Fundus examination of her right eye showed shrinkage of the choroidal tuberculoma with more well-defined margin (Figure 6). The patient was asked to continue the antitubercular therapy for up to 9 months, and followed up once a month.

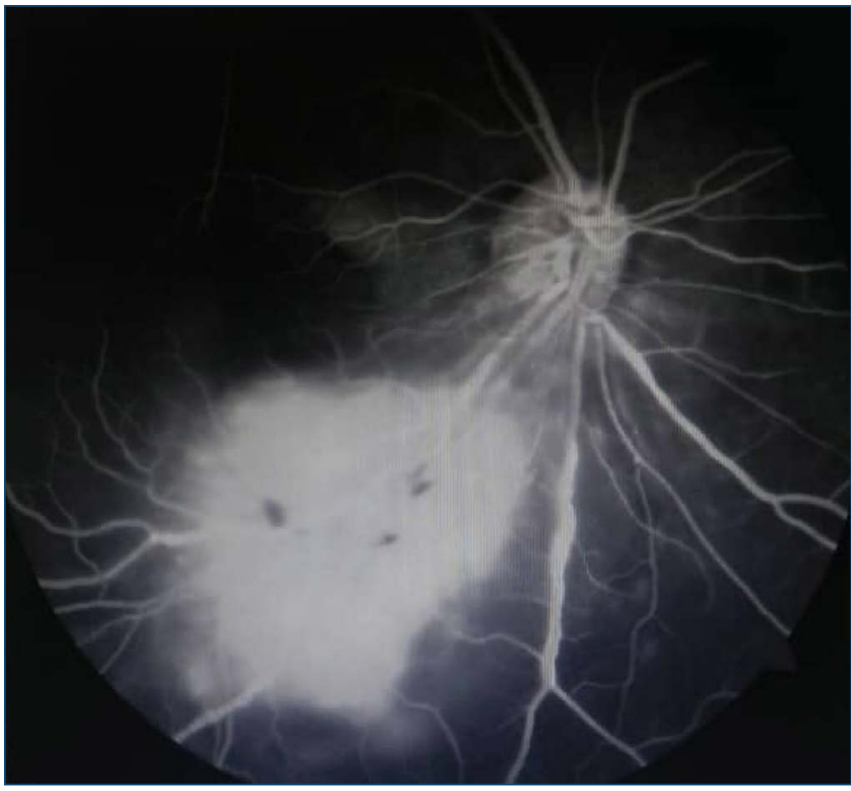

Figure 5. Late phase showed increased hyperfluorescence, but no increase in size suggestive of pooling of dye within tuberculoma.

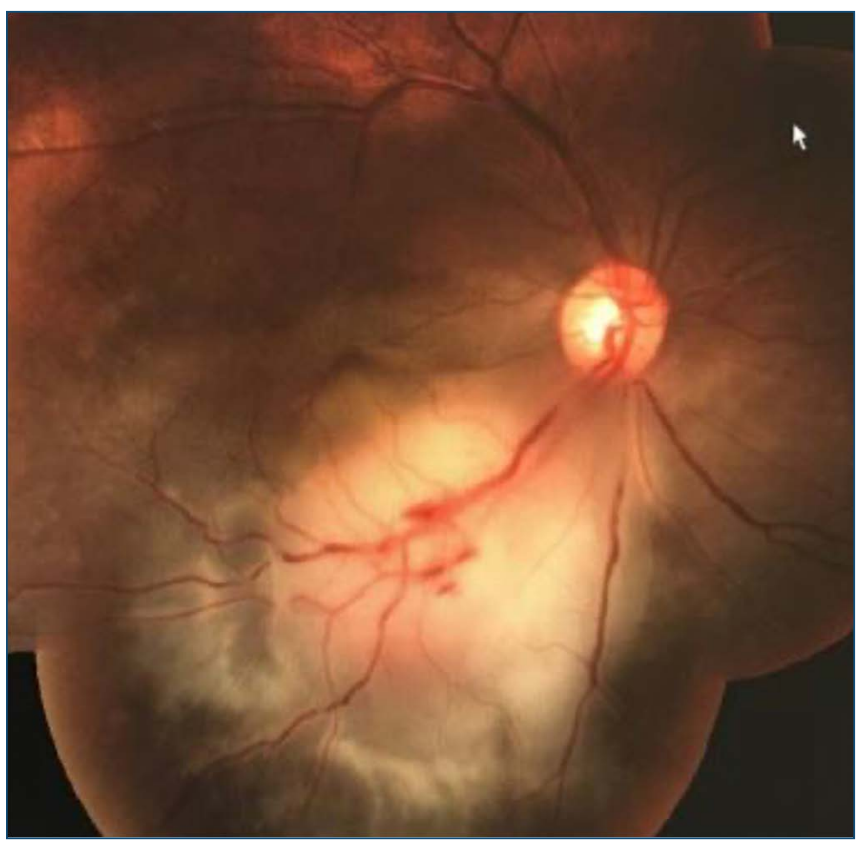

Figure 6. A magnified image of the tuberculoma with more defined margins, after 4 weeks of antituberculosis treatment.

\section{DISCUSSION}

Tuberculosis is an airborne communicable disease that most commonly involves the lungs. Nearly one-third of the world population is latently infected by TB, and more than 9 million new cases are diagnosed each year; in that, 95\% in developing countries. Ocular TB may not be associated with the clinical evidence of pulmonary TB; up to $60 \%$ of patients with evidence of extrapulmonary TB may not have diagnosed pulmonary TB. Ocular TB may be an 
initial presentation of extrapulmonary dissemination of infection, and is a rare event ( $1 \%$ of all cases of TB).

In this case, the patient was diagnosed as presumed ocular TB, based on the history of pulmonary TB contact with a relative, significant Mantoux test measuring over $15 \mathrm{~mm}$, and response to antituberculosis treatment with no recurrences. For presumed intraocular TB, El-Asrar et al. and Gupta et al., in their reviews of intraocular TB, have defined presumed intraocular TB as the following: ocular findings consistent with possible intraocular TB with no other cause of uveitis suggested by history of symptoms, or ancillary testing; strongly positive tuberculin skin test results ( $\geq 15$ $\mathrm{mm}$ area of induration/necrosis), radiological evidence of healed or active tubercular lesion in the chest or evidence of confirmed active extrapulmonary TB, either by microscopic examination or by culture from affected tissue; response to antituberculosis therapy with absence of recurrences. ${ }^{(3,4)}$

Multiple choroidal tubercles have been reported as the most common intraocular manifestation of tubercular posterior uveitis. Less commonly, intraocular TB may present as a large tuberculoma, i.e., a solitary yellowish or greyish white large lesion, generally located in the posterior pole. (5) Rapid multiplication of the bacilli within a tuberculoma can cause tissue destruction through liquefactive necrosis, thus forming a surrounding exudative retinal detachment. ${ }^{(6)}$ This case has shown only a large solitary choroidal tuberculoma with no exudative retinal detachment.

Laboratory workup, including tests to rule out other causes of uveitis, was performed. This is shown in other infective disease screening, such as syphilis serology, toxoplasma serology, viral serology, and all results were negative.

Other tests, such as purified protein derivative (PPD) skin testing and chest X-ray and/or chest computed tomography, were done in this case as well. A positive PPD skin test indicates prior exposure and not necessarily active infection. Half of patients with extrapulmonary involvement can have a negative chest $\mathrm{x}$-ray and 20\% may have a negative PPD skin test..$^{(7)}$ In recent years, measurement of interferon gamma by QuantiFERON-TB ${ }^{\circledR}$ or enzyme-linked immunosorbent spot has become a new technique to diagnose TB infection. QuantiFERON-TB ${ }^{\circledR}$ is an indirect test for Mycobacterium tuberculosis infection and has higher specificity than PPD, since it is unaffected by previous BCG. ${ }^{(8)}$

Intraocular fluid analysis or tissue biopsy would make a definitive diagnosis; however, this is not always available. Diagnosis can be made with nucleic acid amplification by either transcription-mediated amplification of 16S RNA or PCR amplification of DNA sequences of the mycobacteria. Chorioretinal biopsy has been used to make diagnosis as well, and can be used with nucleic acid amplification techniques.

The FFA reveals early hyperfluorescence of active choroidal lesions and late leakage. Cicatricial lesions show early blocked hyperfluorescence with late staining. Ultrasonography reveals moderate to low internal reflectivity of large tuberculomas and can distinguish them from intraocular malignancy. The FFA was performed in this case.

Systemic treatment with the first-line combination regimen comprising isoniazid, rifampicin, pyrazinamide, and ethambutol, for a total of 6 to 12 months, has been accepted as standard therapy. If there is evidence of a clinical response to treatment after the 2-month initiation phase, a 4-month consolidation phase should follow using two first-line drugs (isoniazid and rifampin). Although symptoms may not resolve completely, especially in advanced disease, a demonstrable reduction in inflammation is expected. ${ }^{(9)}$ The purpose of using systemic steroid in this case is to reduce the inflammatory damage that can occur to the choroid, due to presence of tuberculoma. The anti-inflammatory dose of systemic steroid is $0.5 \mathrm{mg} / \mathrm{kg} /$ day, and is often initiated after one week of antituberculosis therapy. This is done to assure the antitubercular agent has achieved its minimal inhibitory concentration prior to steroid initiation. In the present case, a favorable response to treatment was demonstrated by shrinking of the tuberculoma, as shown in the fundus picture at 4 weeks.

\section{REFERENCES}

1. Paul Chan RV, Lai TY, Pettey F, Patel S, editors. Ocular tuberculosis (TB)Asia Pacific. American Academy of Ophthalmology; 2004 [cited 2021 Mar 8]. Available from: https://www.aao.org/topic-detail/ocular-tuberculosistb-asia-pacific

2. Bodaghi B, LeHoang P. Ocular tuberculosis. Curr Opin Ophthalmol. 2000;11(6):443-8.

3. Gupta V, Gupta A, Rao NA. Intraocular tuberculosis-an update. Surv Ophthalmol. 2007;52(6):561-87.

4. El-Asrar AM, Abouammoh M, Al-Mezaine HS. Tuberculous uveitis. Middle East Afr J Ophthalmol. 2009;16(4):188-201.

5. Mansour AM, Haymond R. Choroidal tuberculomas without evidence of extraocular tuberculosis. Graefes Arch Clin Exp Ophthalmol. 1990;228(4):382-3.

6. Arej N, Fadlallah A, Chelala E. Choroidal tuberculoma as a presenting sign of tuberculosis. Int Med Case Rep J. 2016;9:365-8.

7. Intraocular Inflammation and Uveitis. Basic and Clinical Science Course. San Francisco (CA): American Academy of Ophthalmology; 2006

8. Kang YA, Lee HW, Yoon HI, Cho B, Han SK, Shim YS, et al. Discrepancy between the tuberculin skin test and the whole-blood interferon gamma assay for the diagnosis of latent tuberculosis infection in an intermediate tuberculosis-burden country. JAMA. 2005;293(22):275661.

9. Zhang M, Zhang J, Liu Y. Clinical presentations and therapeutic effect of presumed choroidal tuberculosis. Retina. 2012;32(4):805-13. 